\title{
Prevalence of Acute Coronary Syndrome and Various Risk Factors in Acute Stroke Patients
}

\author{
Muhammad Humayoun Rashid ${ }^{1,2}$, Ghulam Yaseen ${ }^{1}$, Umar Ghaffar ${ }^{1}$, Ahmad Ali Khan ${ }^{3,1}$, Ahmad Kabir ${ }^{4}$, \\ ${ }^{1}$, Azka Aisha ${ }^{1}$, Aqsa Komel ${ }^{1}$ \\ 1. Internal Medicine, Nishtar Medical University, Multan, PAK 2. Cardiology, Ch. Pervaiz Elahi Institute of Cardiology, \\ Multan, PAK 3. Pathology, Ch. Pervaiz Elahi Institute of Cardiology, Multan, PAK 4. Anesthesia \& Critical Care, Ch. \\ Pervaiz Elahi Institute of Cardiology, Multan, PAK
}

Corresponding author: Muhammad Humayoun Rashid, humayounchaudhry@gmail.com

\section{Abstract \\ Background}

Stroke is the second leading cause of death worldwide after acute coronary syndrome (ACS). Both diseases share many risk factors such as hypertension, diabetes, dyslipidemia, and smoking. Patients who experience acute coronary syndrome are at heightened risk of recurrent ischemic events such as ischemic strokes, one of the most feared cardiovascular events because of the risk of long-term disability. We tried to estimate the prevalence of underlying ACS among patients with acute stroke.

\section{Methods}

This cross-sectional study was done at the CPE Institute of Cardiology, Pakistan, and Nishtar Medical University and Hospital, Pakistan. A total of 160 acute stroke cases were selected by consecutive sampling technique and questionnaire forms were filled. Detailed history, investigations, and physical examinations were done. The primary outcome was the prevalence of ACS and secondary outcomes were the prevalence of hypertension, smoking, dyslipidemia, diabetes mellitus, and previous history of stroke in stroke patients.

\section{Results}

Most of the patients that presented to us were above 50 years of age with the mean age of 62 years ( $S D=9.23$ years). Male predominance was seen with a total of $115(72 \%)$ cases. Out of 160 patients, $91(57 \%, \mathrm{p}<0.05)$ had underlying ACS, with 45 cases (49\%) with unstable angina, 20 (22\%) with non-ST-elevation myocardial infarction (NSTEMI), and 26 (29\%) with ST-elevation myocardial infarction (STEMI). Prevalence of risk factors in 160 cases were, hypertension (101, 63\%), lack of exercise (91, 57\%), smoking (70, 44\%), diabetes mellitus $(61,38 \%)$, dyslipidemia $(50,31 \%)$. All these results were statistically significant $(\mathrm{p}<0.05)$. Prevalence of obesity $(35,22 \%)$ and previous stroke history $(19,12 \%)$ was statistically insignificant $(p>$ $0.05)$.

Received 07/14/2020 Review began 07/16/2020 Review ended 08/02/2020 Published 08/04/2020

() Copyright 2020 Rashid et al. This is an open access article distributed under the terms of the Creative Commons Attribution License CC-BY 4.0., which permits unrestricted use, distribution, and reproduction in any medium, provided the original author and source are credited.

\section{Conclusion}

Acute coronary syndrome is still frequently present in patients with acute stroke. The need of the hour is to manage ACS efficiently so that its deadly complications such as stroke can be prevented.

Categories: Cardiology, Internal Medicine, Neurology

Keywords: acute coronary syndrome, stroke, hypertension, dyslipidemia, diabetes mellitus, smoking, obesity

\section{Introduction}

Stroke is the second leading cause of death worldwide after acute coronary syndrome (ACS). Both diseases share a lot of risk factors such as hypertension, diabetes, dyslipidemia, and smoking. ACS is diagnosed when patients present with unstable angina, non-ST-elevation myocardial infarction (NSTEMI), or ST-elevation myocardial infarction (STEMI). Such patients have a wide spectrum of risks for death and cardiovascular ischemic events. Careful risk assessment of ACS patients helps clinicians to determine prognosis and may, therefore, be useful in guiding management and providing valuable information to patients [1,2]. To be clinically practical, a risk stratification model must be straightforward and use clinical risk factors that are readily ascertainable at hospital presentation.

ACS and some subtypes of ischemic stroke share similar pathophysiology, including inflammation and the development of atherosclerosis. The acute coronary syndrome can itself be a risk factor for stroke due to thromboembolism, or ischemia-induced atrial fibrillation, or stasis of blood. Several scoring methods, including GRACE (Global Registry of Acute Coronary Events) [3], TIMI (Thrombolysis in Myocardial Infarction) [4], and PURSUIT (Platelet glycoprotein IIb/IIIa in Unstable angina: Receptor Suppression Using Integrilin Therapy) [5], are developed to assess ACS patients who are at risk of developing complications so 
that they can be managed efficiently and timely to reduce disability. Stroke is a rare but serious complication of acute coronary syndrome. At present, no specific score exists to identify patients at higher risk of developing stroke after ACS. The present study aims to check how many of the stroke patients presenting in the emergency and outpatient department have the underlying acute coronary syndrome.

\section{Materials And Methods \\ Study design}

This cross-sectional study took place at the CPE Institute of Cardiology Multan, Pakistan, and Nishtar Medical University and Hospital Multan, Pakistan. Ethics committee approval was obtained from both the hospitals. The non-probability consecutive sampling technique was used. A sample of 160 acute stroke cases, from January 2020 to June 2020, presenting in emergency, outpatient and inpatient departments, was taken on board to fill a questionnaire form. Inclusion criteria were age 30-80, either gender and diagnosed stroke either hemorrhagic or ischemic. Exclusion criteria were patients with age less than 30 years or greater than 80 years, patients with chronic obstructive pulmonary disease, coagulation disorders, antiphospholipid syndrome, HIV, recent surgical operations, pregnancy, and malignancy.

American Heart Association definitions for ACS subtypes were used. Unstable angina is defined as anginal pain that occurs in a resting state with neither ST-segment elevation, nor elevated biomarkers of myocardial ischemia. NSTEMI is defined as chest pain with elevated biomarkers of myocardial ischemia (cardiac enzymes, troponins) but no ST-segment elevations. STEMI is defined as chest pain with elevated biomarkers of myocardial ischemia and ST-segment elevations or new-onset left bundle branch block. Stroke was defined as neurological dysfunction caused by an ischemic or hemorrhagic event with residual symptoms at least $24 \mathrm{~h}$ after onset or leading to death [6].

\section{Outcomes}

The primary outcome of our study was to know the prevalence of acute coronary syndrome in stroke patients on retrospective analysis, and which type of acute coronary syndrome was most prevalent among them. As our secondary outcomes, characteristics of the patients such as age, gender, diabetes mellitus, hypertension, dyslipidemia, smoking, and previous stroke episode were noted down and their prevalence in stroke patients was taken into consideration.

\section{Data collection}

A total of 160 stroke patients that met our inclusion criteria were selected by a convenience sampling method using consecutive sampling technique and they were properly educated about the study. Informed consent was taken from all of them. Stroke type was diagnosed mostly by brain computed tomography (CT) scan or magnetic resonance imaging to detect either infarction or hemorrhage (intracerebral, subdural, or subarachnoid). A detailed history and physical examinations were done. Each patient underwent a series of investigations including CT scan brain, ECG, transthoracic echocardiography, HbA1C, lipid profile, and carotid Doppler studies. Their previous records of the acute coronary syndrome were checked thoroughly. Information regarding the use of ACS medication, any ACS intervention that has been done previously, hospital admissions, previous ECGs, cardiac biomarker reports, and echocardiography reports, was taken. Our team collaborated and divided the tasks of history taking, examination, following the investigations, and gathering data of their previous hospital registrations.

\section{Data analysis}

Data was entered and analyzed in Microsoft Office Excel 2016 and SPSS Statistics version 19 (IBM Corp., Armonk, NY). Mean and standard deviations were calculated for continuous variables such as age, and the student's t-test was used for analysis. Frequencies and percentages were calculated for categorical variables such as ACS, diabetes mellitus, hypertension, gender, dyslipidemia, smoking, and previous stroke or transient ischemic attack (TIA). The Chi-square test was used for analysis. All the data was compiled in the form of flow charts, spreadsheets, and histograms.

\section{Results}

The mean age of the patients was 62 years \pm 9.23 years (standard deviation). The minimum age of stroke presentation was 34 years and the maximum age of presentation was 78 years as shown in Table 1 . Out of 160 cases that were recruited in the study, 117 (73\%) were above 50 years of age and 43 (27\%) were under the age of 50 years at the time of presentation as shown in Figure 1. 


\section{Cureus}

\begin{tabular}{|c|c|c|c|c|}
\hline & MINIMUM & MAXIMUM & MEAN & SD \\
\hline AGE & 34 & 78 & 62 & \pm 9.23 years \\
\hline
\end{tabular}

TABLE 1: Age distribution

SD: Standard deviation

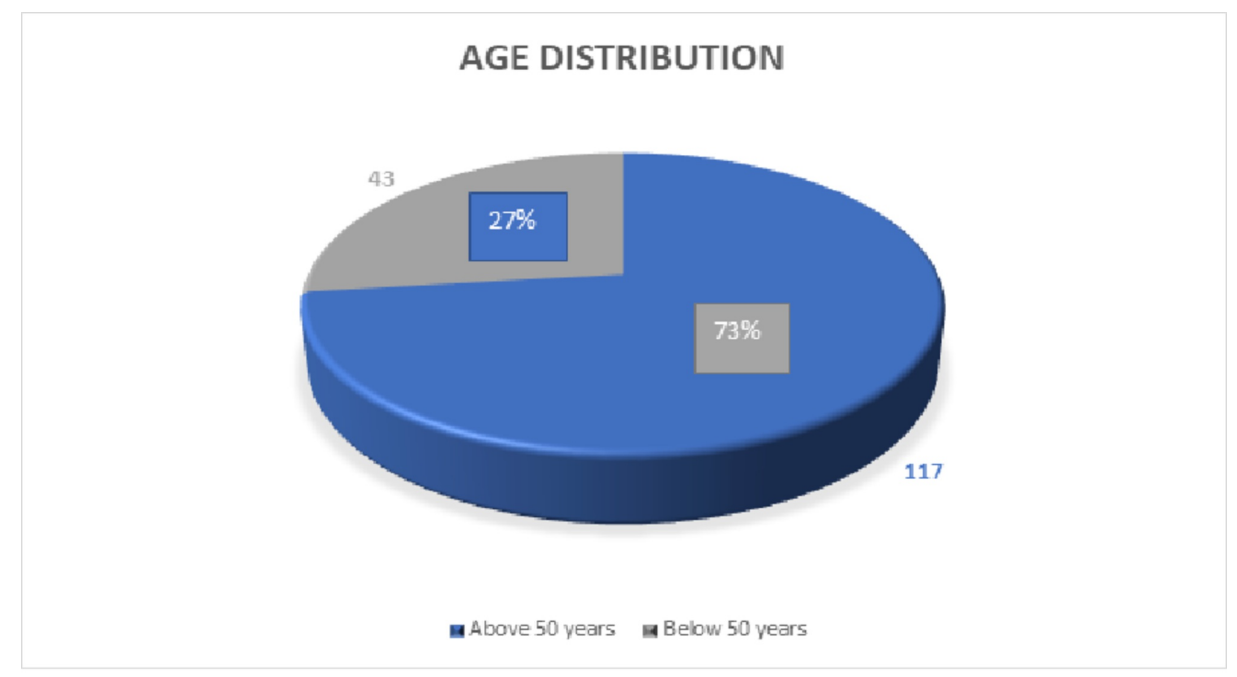

FIGURE 1: Age distribution pie chart

Non-probability consecutive sampling technique was followed. Out of 160 cases, 115 (72\%) male patients, and 45 (28\%) female patients presented to us. This showed male predominance in stroke cases concluding that male gender itself possesses more risk for stroke occurrence. The results are displayed in Figure 2.

\section{GENDER DISTRIBUTION}

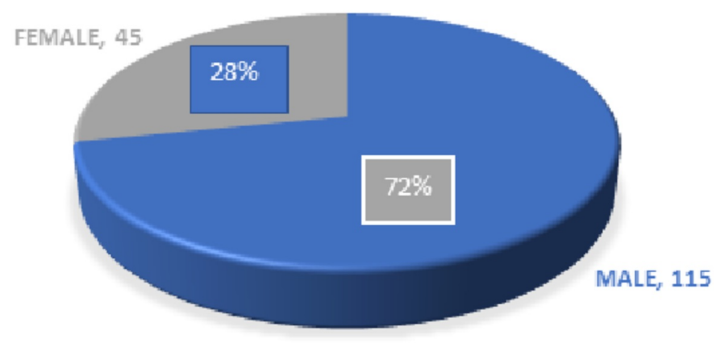

a MALE

\section{FIGURE 2: Gender distribution pie chart}

As our primary outcome we analyzed that out of 160 cases, 91 cases i.e. $57 \%(p<0.05)$ had underlying acute coronary syndrome associated with stroke, and 69 cases i.e. $43 \%(\mathrm{p}<0.05)$ had no underlying acute coronary syndrome in an association. Among the 91 ACS cases, 45 (49\%) had unstable angina, 20 (22\%) had NSTEMI, and 26 (29\%) had STEMI. The data is compiled in Table 2 and presented graphically in Figure 3. 


\section{Cureus}

\begin{tabular}{|c|c|c|c|c|}
\hline STROKE PATIENTS & NO ACS & & ACS & \\
\hline$(n=160)$ & & UNSTABLE ANGINA & NSTEMI & STEMI \\
\hline Number & 69 & 45 & 20 & 26 \\
\hline Percentage & $43 \%$ & $49 \%$ & $22 \%$ & $29 \%$ \\
\hline
\end{tabular}

\section{TABLE 2: Prevalence of ACS in acute stroke}

ACS: Acute coronary syndrome; NSTEMI: Non-ST-elevation myocardial infarction; STEMI: ST-elevation myocardial infarction.

FREQUENCY OF ACS IN STROKE

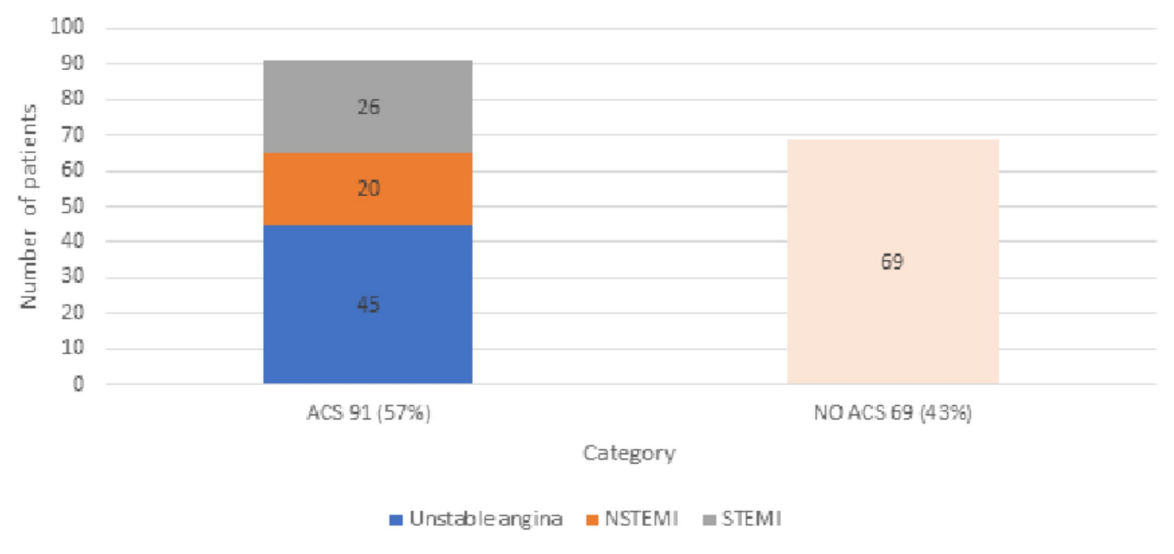

\section{FIGURE 3: Prevalence of ACS in acute stroke}

ACS: Acute coronary syndrome; NSTEMI: Non-ST-elevation myocardial infarction; STEMI: ST-elevation myocardial infarction.

We studied the prevalence of risk factors that were present in the stroke patients that were included in the study. Questionnaires of our study included questions about the risk factors that are associated with stroke according to the American Academy of Neurology and the American Heart Association and were filled by the attendants of the patients mostly. This showed that among 160 stroke patients, 101 (63\%) had hypertension, 91 (57\%) had lack of exercise, 70 (44\%) had smoking history, 61 (38\%) had diabetes mellitus, 50 (31\%) had dyslipidemia, 35 (22\%) were obese and 19 (12\%) had the previous history of stroke or TIA. These results are displayed in the following Figure 4.

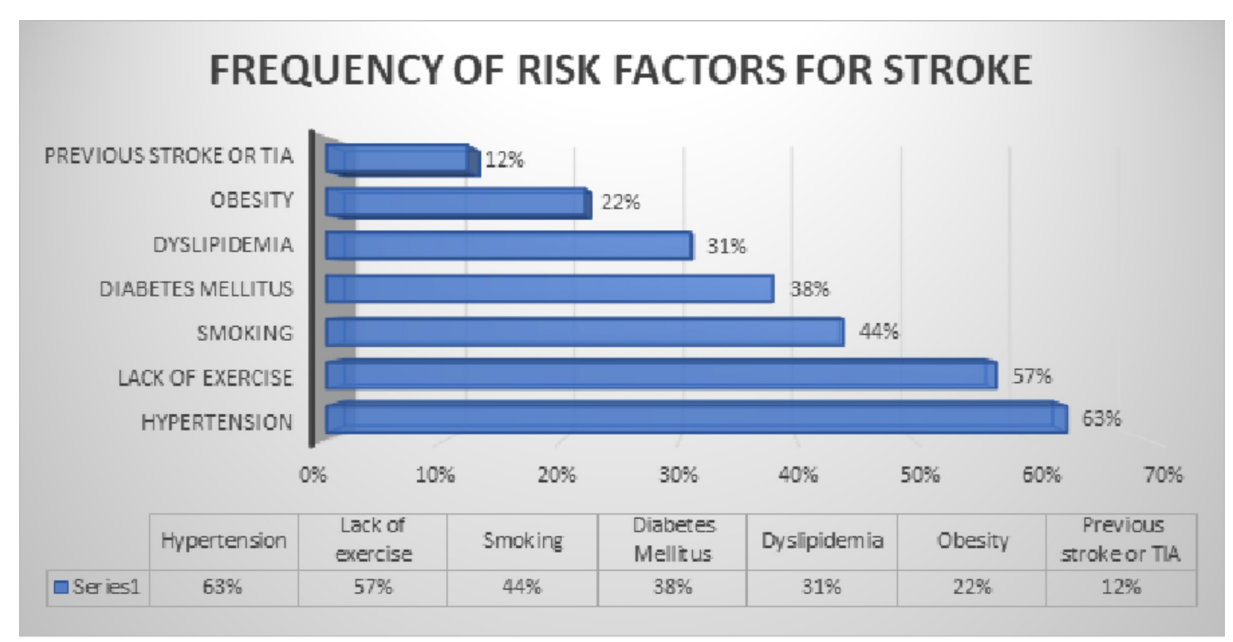


Stratification of hypertension, lack of exercise, smoking, diabetes mellitus, dyslipidemia, obesity, and previous stroke or TIA with stroke, and acute coronary syndrome is given in Table 3. This table shows that hypertension is strongly associated with both stroke and ACS with $63 \%$ and $55 \%(\mathrm{p}<0.05)$ occurrence respectively and so do lack of exercise with $57 \%$ and $69 \%(p<0.05)$, respectively.

\begin{tabular}{|c|c|c|c|c|c|c|}
\hline RISK FACTORS & STATUS & STROKE PATIENTS (160) & PERCENTAGE & ACS PATIENTS (91) & PERCENTAGE & P-VALUE \\
\hline \multirow[t]{2}{*}{ Hypertension } & Present & 101 & $63 \%$ & 50 & $55 \%$ & $<0.05$ \\
\hline & Absent & 59 & $37 \%$ & 41 & $45 \%$ & \\
\hline \multirow[t]{2}{*}{ Lack of exercise } & Present & 91 & $5 / \%$ & 63 & $69 \%$ & $<0.05$ \\
\hline & Absent & 69 & $43 \%$ & 28 & $31 \%$ & \\
\hline \multirow[t]{2}{*}{ Smoking } & Present & 70 & $44 \%$ & 32 & $35 \%$ & $<0.05$ \\
\hline & Absent & 90 & $56 \%$ & 59 & $65 \%$ & \\
\hline \multirow[t]{2}{*}{ Diabetes mellitus } & Present & 61 & $38 \%$ & 36 & $40 \%$ & $<0.05$ \\
\hline & Absent & 99 & $62 \%$ & 55 & $60 \%$ & \\
\hline \multirow[t]{2}{*}{ Dyslipidemia } & Present & 50 & $31 \%$ & 25 & $27 \%$ & $<0.05$ \\
\hline & Absent & 110 & $69 \%$ & 66 & $73 \%$ & \\
\hline \multirow[t]{2}{*}{ Obesity } & Present & 35 & $22 \%$ & 16 & $18 \%$ & $>0.05$ \\
\hline & Absent & 125 & $78 \%$ & 75 & $82 \%$ & \\
\hline \multirow[t]{2}{*}{ Previous stroke or TIA } & Present & 19 & $12 \%$ & 6 & $7 \%$ & $>0.05$ \\
\hline & Absent & 141 & $88 \%$ & 85 & $93 \%$ & \\
\hline
\end{tabular}

\section{TABLE 3: Prevalence of various risk factors in acute stroke}

TIA: Transient ischemic attack

\section{Discussion}

We did a cross-sectional study to estimate the prevalence of acute coronary syndrome in patients with acute stroke onset. Patients who experience acute coronary syndrome are at heightened risk of recurrent ischemic events [7-9]. These events are not limited to the coronary vascular bed but also include ischemic strokes, one of the most feared cardiovascular events because of the risk of long-term disability [10]. Hence, we tried to estimate the prevalence of underlying ACS among patients with acute stroke and the result came out to be $57 \%(\mathrm{p}<0.05)$ and was statistically significant. It shows that ACS is still very much prevalent in stroke. Apart from the fact that the pathophysiology and most of the risk factors for stroke and ACS are similar, ACS itself is a risk factor for ischemic stroke mostly due to stasis of blood in ventricles, ACS induced atrial fibrillation and turbulence of blood can cause clot formation in chambers of the heart which can dislodge and travel through major arteries towards brain causing a stroke. Studies also indicate that myocardial infarction is a major risk factor for acute stroke onset $[11,12]$. The efficient management of acute coronary syndrome is required so that such deadly complications like a stroke can be reduced.

Most of the cases that presented to us were above the age of 50 years. And the mean age came out to be 62 years. It is explained partly by the fact that with age the vessels become less elastic and more fragile, and also that the aged people have increased number of co-morbidities like hypertension, diabetes, and dyslipidemias. Similarly, the male gender was found to have a higher incidence of stroke with up to the fourfold increased risk of stroke. Hence age and gender are uncontrollable risk factors and independent predictors of acute stroke onset. Most of the risk factors identified in this study correlated with those that have been identified in other studies. Risk factors that have appeared repeatedly in other studies include older age, history of a previous stroke, hypertension, diabetes mellitus, dyslipidemia, carotid and peripheral vascular disease, obesity, and atrial fibrillation. It is interesting to note that most of the risk factors for 
stroke in the cases we studied, closely mirror those contained in the CHA2DS2-VASc scoring system, which was derived and validated as a tool for stroke risk stratification in patients with atrial fibrillation [13-15]. Atrial fibrillation (AF) is associated with a $\approx 5$-fold increased risk of ischemic stroke, and stroke prevention is a major priority in the clinical management of AF. When compared with control/placebo, oral anticoagulation (OAC) therapy reduces the risk of stroke by $64 \%$, and the risk of death by $26 \%$ [16]. A systematic review and meta-analysis on CHA2DS2-VASc risk factors as predictors of stroke after acute coronary syndrome also studied that they have statistically significant predictive value for stroke onset in the patients having underlying ACS [17].

Our study indicates that both hypertension and diabetes mellitus are common in patients with stroke and ACS. Hypertension was $63 \%$ prevalent in stroke patients and $55 \%$ in ACS patients. Similarly, diabetes mellitus was $38 \%$ prevalent in stroke and $40 \%$ in ACS patients. These risk factors cause atherosclerosis and arteriosclerosis which can lead to turbulence of blood flow, plaque formation, and rupture leading to thrombus formation and ischemic events, commonly in heart and brain [18-21]. Dyslipidemia is also found commonly in stroke and ACS patients. Our study showed $31 \%$ and $27 \%$ prevalence, respectively. A large randomized controlled trial on 3216 patients showed that adults with both high triglyceride (TG) and low high-density lipoprotein cholesterol (HDL-C), particularly those with diabetes, have increased risks of coronary heart disease and stroke. In particular, those with an LDL-C level of $\geqslant 130 \mathrm{mg} / \mathrm{dL}$ may have an increased risk of incident stroke [22]. Lipid-lowering therapy with statins has been shown to result in a $22 \%$ reduction in major vascular events per $1 \mathrm{mmol} / \mathrm{L}$ reduction in low-density lipoprotein cholesterol (LDL-C), including a $21 \%$ relative risk reduction in ischemic stroke per $1 \mathrm{mmol} / \mathrm{L}$ reduction in LDL-C.

Cigarette smoking is an important risk factor for all-cause mortality as well as vascular disease mortality $[23,24]$. Many prospective cohort studies conducted in Western populations, as well as Asian populations, have indicated a strong and independent relationship between cigarette smoking and risk of stroke [25-28]. Our study also showed a high prevalence of smoking in stroke patients which is $44 \%$. The prevalence of obesity and previous stroke history came out to be $22 \%$ and $12 \%$ and the results were statistically insignificant. Maybe because the study sample was not high enough to prove the association. Whereas, in an analysis adjusted for age, sex, race, and smoking, obesity was associated with a $65 \%$ increased risk for ischemic stroke (odds ratio, 1.65; 95\% confidence interval, 1.33-2.04). Some people believe that treating obesity decreases the risk of stroke, while some say that treating the consequences of obesity such as diabetes and hypertension imparts the benefits [29]. These risk factors are still debatable and highly researched topics in cardiology and neurology.

\section{Conclusions}

Acute coronary syndrome is still frequently present in patients with acute stroke. Older age and male gender are independent predictors of stroke occurrence. Risk factors such as hypertension, dyslipidemia, lack of exercise, smoking, and diabetes mellitus are also increasingly prevalent. The need of the hour is to manage ACS efficiently so that its deadly complications such as stroke can be prevented from occurring.

\section{Additional Information}

\section{Disclosures}

Human subjects: Consent was obtained by all participants in this study. Nishtar Medical University issued approval 36580/950. Ethics committee and research department of Nishtar Medical University was informed about the research and approval was taken. Animal subjects: All authors have confirmed that this study did not involve animal subjects or tissue. Conflicts of interest: In compliance with the ICMJE uniform disclosure form, all authors declare the following: Payment/services info: All authors have declared that no financial support was received from any organization for the submitted work. Financial relationships: All authors have declared that they have no financial relationships at present or within the previous three years with any organizations that might have an interest in the submitted work. Other relationships: All authors have declared that there are no other relationships or activities that could appear to have influenced the submitted work.

\section{References}

1. Boden H, Van der Hoeven BL, Karalis I, Schalij MJ, Jukema JW: Management of acute coronary syndrome: achievements and goals still to pursue. Novel developments in diagnosis and treatment. JIM. 2012, 271:521536. 10.1111/j.1365-2796.2012.02533.x

2. Nakatani D, Sakata Y, Suna S, et al.: Incidence, predictors, and subsequent mortality risk of recurrent myocardial infarction in patients following discharge for acute myocardial infarction. Circulation J. 2013, 77:439-446. 10.1253/circj.CJ-11-1059

3. Fox KA, Dabbous OH, Goldberg RJ, et al.: Prediction of risk of death and myocardial infarction in the six months after presentation with acute coronary syndrome: prospective multinational observational study (GRACE). BMJ. 2006, 333:1091. 10.1136/bmj.38985.646481.55

4. Antman EM, Cohen M, Bernink PJ, et al.: The TIMI risk score for unstable angina/non-ST elevation MI: a method for prognostication and therapeutic decision making. JAMA. 2000, 284:835-842. 10.1001/jama.284.7.835

5. Boersma E, Pieper KS, Steyerberg EW, et al.: Predictors of outcome in patients with acute coronary 
syndromes without persistent ST-segment elevation: results from an international trial of 9461 patients. Circulation. 2000, 101:2557-2567. 10.1161/01.CIR.101.22.2557

6. Felker GM, Pang PS, Adams KF, et al.: Clinical trials of pharmacological therapies in acute heart failure syndromes: lessons learned and directions forward. Circulation: Heart Failure. 2010, 3:314-325.

10.1161/CIRCHEARTFAILURE.109.893222

7. Mooe T, Olofsson BO, Stegmayr B, Eriksson P: Ischemic stroke: impact of a recent myocardial infarction . Stroke. 1999, 30:997-1001. 10.1161/01.STR.30.5.997

8. Bhatt DL, Eagle KA, Ohman EM, et al.: Comparative determinants of 4-year cardiovascular event rates in stable outpatients at risk of or with atherothrombosis. JAMA. 2010, 304:1350-1357. 10.1001/jama.2010.1322

9. Witt BJ, Brown RD Jr, Jacobsen SJ, Weston SA, Yawn BP, Roger VL: A community-based study of stroke incidence after myocardial infarction. Ann Intern Med. 2005, 143:785-792. 10.7326/0003-4819-143-11200512060-00006

10. Mozaffarian D, Benjamin EJ, Go AS, et al.: Executive summary: heart disease and stroke statistics-2016 update: a report from the American Heart Association. Circulation. 2016, 133:447-454. 10.1161/CIR.0000000000000366

11. Komrad MS, Coffey CE, Coffey KS, McKinnis R, Massey EW, Califf RM: Myocardial infarction and stroke. Neurology. 1984, 34:1403. 10.1212/WNL.34.11.1403

12. AlSaeed W, Al-Zakwani I, Panduranga P, Zubaid M, Rashed W, Brady PA: Incidence, predictors, and mortality of in-hospital stroke after acute coronary syndrome in the Middle East. Ann Clin Cardiol. 2020, 2:13-18. 10.4103/ACCJ.ACCJ_4_20

13. Lip GY, Nieuwlaat R, Pisters R, Land DA, Crijns H: Refining clinical risk stratification for predicting stroke and thromboembolism in atrial fibrillation using a novel risk factor-based approach: the Euro heart survey on atrial fibrillation. Chest. 2010, 137:263-272. 10.1378/chest.09-1584

14. Friberg L, Rosenqvist M, Lip GY: Evaluation of risk stratification schemes for ischaemic stroke and bleeding in 182678 patients with atrial fibrillation: the Swedish Atrial Fibrillation cohort study. Eur Heart J. 2012, 33:1500-1510. 10.1093/eurheartj/ehr488

15. Kim TH, Yang PS, Uhm JS, et al.: CHA2DS2-VASc score (congestive heart failure, hypertension, age $\geqslant 75$ [doubled], diabetes mellitus, prior stroke or transient ischemic attack [doubled], vascular disease, age 65-74, female) for stroke in Asian patients with atrial fibrillation: a Korean nationwide sample cohort study. Stroke. 2017, 48:1524-1530. 10.1161/STROKEAHA.117.016926

16. Hart RG, Pearce LA, Aguilar MI: Meta-analysis: antithrombotic therapy to prevent stroke in patients who have nonvalvular atrial fibrillation. Ann Intern Med. 2007, 146:857-867. 10.7326/0003-4819-146-12200706190-00007

17. Guerra F, Scappini L, Maolo A, Campo G, Pavasini R, Shkoza M, Capucci A: CHA2DS2-VASc risk factors as predictors of stroke after acute coronary syndrome: a systematic review and meta-analysis. Eur Heart J Acute Cardiovasc Care. 2018, 7:264-274. 10.1177/2048872616673536

18. Alloubani A, Saleh A, Abdelhafiz I: Hypertension and diabetes mellitus as a predictive risk factors for stroke . Diabetes Metab Syndr. 2018, 12:577-584. 10.1016/j.dsx.2018.03.009

19. Madmoli M, Saeidilandi M, Latifinasab R, Fatehimoghaddam S, Mobarez F, Darabiyan P: Hypertension and risk of acute coronary syndrome (ACS) in patients with ACS: a study on 926 patients with ACS. IJAM. 2019, 10:22-26.

20. Esteghamati A, Abbasi M, Nakhjavani M, Yousefizadeh A, Basa AP, Afshar H: Prevalence of diabetes and other cardiovascular risk factors in an Iranian population with acute coronary syndrome. Cardiovasc Diabetol. 2006, 5:15. 10.1186/1475-2840-5-15

21. Turin TC, Okamura T, Afzal AR, et al.: Hypertension and lifetime risk of stroke. Int J Hypertens. 2016, 34:116-122. 10.1097/HJH.0000000000000753

22. Lee JS, Chang PY, Zhang Y, Kizer JR, Best LG, Howard BV: Triglyceride and HDL-C dyslipidemia and risks of coronary heart disease and ischemic stroke by glycemic dysregulation status: the strong heart study. Diabetes Care. 2017, 40:529-537. 10.2337/dc16-1958

23. Lopez AD, Mathers CD, Ezzati M, Jamison DT, Murray CJL: Global and regional burden of disease and risk factors, 2001: systematic analysis of population health data. Lancet. 2006, 367:1747-1757. 10.1016/S01406736(06)68770-9

24. Strong K, Mathers C, Bonita R: Preventing stroke: saving lives around the world . Lancet Neurol. 2007, 6:182187. 10.1016/S1474-4422(07)70031-5

25. Wolf PA, D'Agostino RB, Kannel WB, Bonita R, Belanger AJ: Cigarette smoking as a risk factor for stroke: the Framingham study. JAMA. 1988, 259:1025-1029. 10.1001/jama.1988.03720070025028

26. Colditz GA, Bonita R, Stampfer MJ, Willett WC, Rosner B, Speizer FE, Hennekens CH: Cigarette smoking and risk of stroke in middle-aged women. N Engl J Med. 1988, 318:937-941. 10.1056/NEJM198804143181501

27. Kelly TN, Gu D, Chen J, et al.: Cigarette smoking and risk of stroke in the Chinese adult population . Stroke. 2008, 39:1688-1693. 10.1161/STROKEAHA.107.505305

28. Mannami T, Iso H, Baba S, et al.: Cigarette smoking and risk of stroke and its subtypes among middle-aged Japanese men and women: the JPHC Study Cohort I. Stroke. 2004, 35:1248-1253. 10.1161/01.STR.0000128794.30660.e8

29. Mitchell AB, Cole JW, McArdle PF, et al.: Obesity increases risk of ischemic stroke in young adults. Stroke. 2015, 46:1690-1692. 10.1161/STROKEAHA.115.008940 